\title{
Coaxiality evaluation based on double material condition
}

\author{
Zhemin Tang ${ }^{\mathrm{a}}$, Meifa Huang ${ }^{\mathrm{a}, *}$, Yonghou Suna, Yanru Zhong ${ }^{\mathrm{b}}$, Yuchu Qin ${ }^{\mathrm{c}}$, Jing Huang ${ }^{\mathrm{a}}$

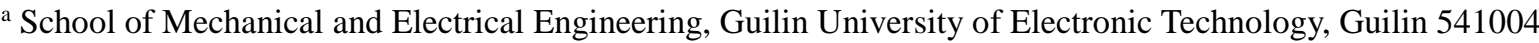 \\ ${ }^{\mathrm{b}}$ School of Computer science and Information Security, Guilin University of Electronic Technology, Guilin 541004 \\ ${ }^{c}$ EPSRC Future Advanced Metrology Hub, Centre for Precision Technologies, School of Computing and Engineering, \\ University of Huddersfield, Huddersfield, HD1 3DH, UK
}

\begin{abstract}
To ensure the assemblability of a workpiece, Coaxiality-DM can be applied to two coaxiality cylinders. According to this specification, the relative orientation and location between an actual datum feature and the derived datum axis are variable. Thus, the Coaxiality-DM tolerance is mainly verified by real gauges, which is costly for small batch production. To develop a proper tool for that production, a evaluation model for Coaxiality-DM tolerance was investigated in this paper. According to the International Organization for Standardization (ISO), the geometry of the real gauge was analyzed, and a virtual gauge model was established. Based on the model, an evaluation method was proposed. Furthermore, an example of a stepped shaft was provided to illustrate the applicability of the proposed method. Finally, a comparative experiment was carried out, and the results revealed that the proposed model could produce more accurate results than the existing mathematical methods.
\end{abstract}

Keywords: Coaxiality; Datum; Error evaluation; Mathematical method; Material requirement.

\section{Introduction}

Size and geometric tolerance are important factors for the proper functionality of any product; therefore, geometric evaluation is essential during and after manufacturing. In any evaluation method, dimensional and geometric tolerances are two important types of information used to describe the geometric qualities of a product. These two types of tolerances could compensate for each other when certain material conditions are applied. In general, three types of material conditions are available: Maximum Material Requirement (MMR), Least Material Requirement (LMR), and Reciprocity Requirement (RPR) [1].

When MMR or LMR is applied, the size tolerance compensates for the geometric tolerance, which results in Maximum Material Virtual Boundaries (MMVBs) or Least Material Virtual Boundaries (LMVBs), respectively. Moreover, RPR could be added to MMR or LMR to allow the geometric tolerance to compensate for the size tolerance. From a geometric point of view, MMVB is the outer boundary that controls the maximum material to ensure the minimum clearance and assemblability, whereas LMVB is the inner boundary that controls the minimum material to ensure the minimum thickness and strength of a product [2].

In order to reduce costs and control certain functions of parts with shafts and holes, material conditions could be applied to a cylinder and its datum cylinder of a coaxiality specification, such as the MMR's application to shaft $b$ and shaft $A$ (Figure.1). For convenience, the specification is termed Coaxiality tolerance with Double Material condition (Coaxiality-DM tolerance). The Coaxiality-DM tolerance establishes two size-fixed virtual cylindrical boundaries: one for the considered cylinder and the other for its datum cylinder.

For example, as shown in Figure.1, virtual hole $b^{\prime}$ is established for considered shaft $b$, and virtual hole $A$ ' is established for datum shaft $A$. According to this specification, the datum reference is derived from the virtual boundary for the datum cylinder, such as axis $c^{\prime}$ derived from virtual hole $A^{\prime}$ (Figure.1). Thus, when an actual datum feature has less or more mass than the applied material condition, Movement Relative to Datum Reference (MRDR) is allowed, and more errors can be compensated. 


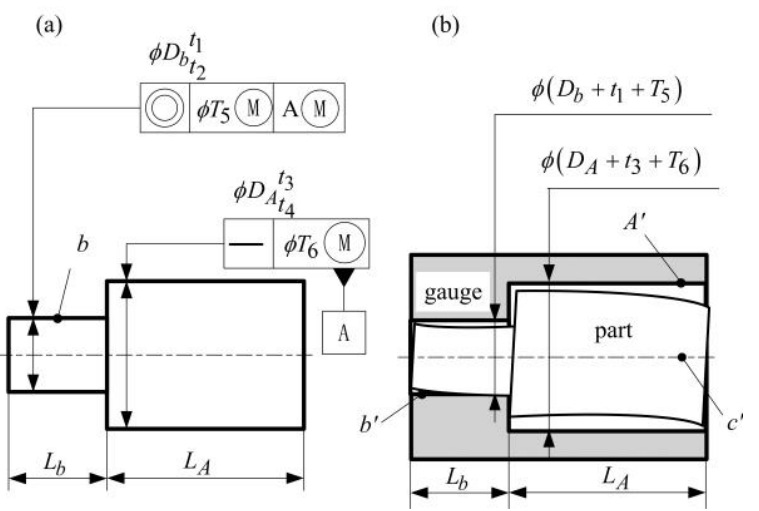

Figure.1. (a) Specification and (b) evaluation of a Coaxiality-DM tolerance

However, MRDR causes a big difference between the evaluation of Coaxiality-DM tolerance and other datum-fixed tolerances. In this case, five major mathematical evaluation approaches are applicable: direct calculation, least squares method (LSM), convex-hull method, geometrical search method, and optimization method.

1. When MRDR of the actual considered feature is allowed, any location error of the considered feature (such as Coaxiality-DM error) varies and cannot be calculated directly.

2. LSM creates an error function for the considered feature before the least squares of measurements are calculated [3, 4]. However, when MRDR is allowed, a function for location error cannot be created directly. Thus, LSM cannot be applied to Coaxiality-DM directly. In addition, an LSM error is not exact according to the International Organization for Standardization (ISO).

3. The convex-hull method establishes the convex-hull of the considered feature before assessing the minimum error by enumeration, thus reducing the data size significantly [5]. However, as Coaxiality-DM error cannot be directly evaluated, the convex-hull method can only be employed to reduce the data size during the evaluation of Coaxiality-DM error.

4. A geometric search method is generally proposed for the error evaluation of one or a few types of features, and it gets the search direction by developed geometric rules. Hence, the geometric search method performs well for certain types of features, whereas the performance is unsatisfactory for others. Currently, it is mainly applied to single simple features, such as the straightness of straight lines [6], sphericity of balls [7], and flatness of planes [6, 8]. However, it is difficult to develop a geometric search method for Coaxiality-DM, which is more complex.

5. If an optimization method is used to evaluate an error as a maximum or a minimum, an error objective function should be established. Currently, a plethora of optimization methods are available, such as the Quasi-Newton method for unconstrained problems; repetitive bracketing method and simplex method for constrained problems [9]; particle swarm optimization and differential evolution algorithm for complex gradient and multi-minimum problems [10, 11]; and inner point method, Active-Set Method (ASM), and Sequential Quadratic Programming (SQP) for medium-scale constrained problems [12]. However, no error function for the considered feature and its datum feature has been created.

It can be seen that establishing the error objective functions for Coaxiality-DM error is key to evaluating Coaxiality-DM tolerance, and some related work has been done in this area:

- A 3D model for axis-symmetric features of Coaxiality-DM tolerance was established based on two assumptions: dimensional, orientation, and location errors were very small; and form error was much smaller [13]. In this model, the form error was ignored, and the orientation and location errors were represented as a linear combination of two translation variables and two rotational variables. Consequently, the method established a concise model, but no form errors were reflected.

- In another study, the T-Map method was adopted to transfer a Coaxiality-DM tolerance into a datum-fixed coaxiality tolerance, which can be measured and evaluated by current mathematical methods [14]. However, the transferred tolerance is stricter than the Coaxiality-DM tolerance, so more parts will be rejected.

Above all, it is noticeable that the error function and optimization based methods for Coaxiality-DM evaluation are still areas of interest in research. Therefore, an accurate method utilizing a virtual gauge is investigated in this paper, which is 
organized as follows:

- In Section.2, the geometric requirements of Coaxiality-DM and its real gauge according to ISO are analyzed.

- In Section.3, a virtual gauge developed from the real gauge is established. Based on the virtual gauge, an optimization model with an error function is established for the considered cylinder and its datum cylinder. Additionally, all necessary steps and formulas for evaluation are given.

- In Section.4, an example of a stepped shaft is provided to illustrate the applicability of the proposed method.

- In Section.5, the proposed method is compared to the T-Map method, and the results indicate the greater accuracy of the proposed method.

\section{Constraints and real gauge}

A Coaxiality-DM tolerance specifies five geometric requirements in ISO:

I. Size requirement: Local sizes of considered and datum cylinders should be between their MMS and LMS. Furthermore, if RPR is added to MMR or LLR, the MMS or the LLS can be broken, respectively.

II. Boundary requirement: The considered cylinder and its datum cylinder should not violate their virtual boundaries. A virtual boundary is an MMVB cylinder for MMR or an LMVB cylinder for LMR.

III. Relative location of boundaries: Virtual boundaries of the considered cylinder and its datum cylinder should be coaxial.

IV. Boundary size of considered cylinder: The virtual boundary size of the considered cylinder should be (a) its MMS plus (for shaft) or minus (for hole) its coaxial tolerance when MMR is applied, or (b) its LMS minus (for shaft) or plus (for hole) its coaxiality tolerance when LMR is applied.

V. Boundary size of datum cylinder: (a) When no geometric tolerance with material requirement is applied to the datum cylinder, its virtual boundary size should be its MMS (for MMR) or LMS (for LMR). (b) When form tolerance with material requirement is applied to the datum cylinder, its virtual boundary size should be (b.1) its MMS plus its geometric tolerance (for shaft with MMR), (b.2) its MMS minus its geometric tolerance (for hole with MMR), (b.3) its LMS plus its geometric tolerance (for hole with LMR), or (b.4) its LMR minus its geometric tolerance (for shaft with LMR).

The size requirement (i.e., requirement I) of a Coaxiality-DM tolerance should be checked regardless of the other four requirements. Additionally, the four boundary-related requirements (i.e., requirements II-V) can be checked by a real gauge, according to ISO. For example, an MMR Coaxiality-DM tolerance applied to the considered shaft $b$ and the datum shaft $A$ in Figure.1.(a) can be checked by the real gauge in Figure.1.(b). The real gauge consists of two coaxial holes, $b^{\prime}$ and $A^{\prime}$, which are as big as the MMVBs of the considered shaft $b$ and the datum shaft $A$, respectively. According to ISO, the part should be able to insert the real gauge.

However, a real gauge can only be used to check certain specifications; therefore, multiple gauges are required to check all the specifications. Moreover, highly accurate gauges are expensive, which may be cost prohibitive for small or medium level production. Virtual parts generated from/for computer-aided design, engineering, or tolerancing cannot be checked by a real gauge. Additionally, the LMR defines the LMVB inside the material, which cannot be checked by a real gauge. In order to increase the flexibility of Coaxiality-DM evaluation, a virtual-gauge-based mathematical method is proposed according to the five geometric requirements of Coaxiality-DM tolerances.

\section{Virtual gauge-based evaluation}

\subsection{General description}

The nominal geometry and dimensions of a mechanical part are generally presented in a computer-aided design model. However, actual real parts cannot be perfectly processed according to the nominal geometry and size. In order to simulate actual real parts, the skin model has been developed [15], where real parts are represented by virtual parts as several continuous functions or discrete geometric features, such as point sets. In this paper, actual real parts and actual virtual parts are collectively called actual parts, for convenience.

Before the evaluation of an actual part, the actual part needs to be represented by extracted features. For each type of actual part mentioned above, certain methods can be employed to obtain extracted features. For an actual real part, the considered cylinder and its datum cylinder can be measured by a coordinate measurement machine, and the measurements are termed Extracted Features of Considered feature (EFC) and Extracted Features of Datum feature (EFD), respectively. Essential positions, such as edges, are required. Since the distribution and reliability of the measurements are discussed in the literature, such as in [16], this paper does not describe them. For a virtual part represented by a continuous function, the coordinates of multiple points on the considered cylinder and its datum cylinder should be collected as EFC and EFD, 
respectively. For a virtual part represented by discrete points, all discrete points of the considered cylinder and its datum cylinder are grouped into EFC and EFD, respectively.

Then, the EFC and EFD replace the considered cylinder and its datum cylinder during the evaluation. Based on this, the size requirement of an actual part can be checked by traditional methods, and the other four boundary-related requirements can be checked by a virtual gauge.

Virtual gauge is an ideal geometric concept that has no solid body and can be reflected by mathematics and displayed through visual techniques. A virtual gauge of a Coaxiality-DM tolerance consists of two coaxial cylinders (i.e., the virtual boundaries of the considered cylinder and its datum cylinder) and reflects requirements III, IV, and V (Figure.2). After a virtual gauge is established, the EFC and EFD can be verified by being placed into their virtual boundaries. Only by placing all extracted features into/outside the virtual gauge can it be proved that the part conforms to requirements II, III, IV, and V. In this paper, the MMVB-coaxial envelope of EFC should be minimum (for MMR) or maximum (for LMR), while EFD should be inside (for MMR) or outside (for LMR) its boundary. The process of meeting these requirements by adjusting the orientations and positions of the extracted features in the virtual gauge is described as a constrained optimization function, and it can be solved by an existing optimization method such as SQP. The result reflects the minimum MMVB-coaxial envelope of EFC, which should be compared with the virtual boundary size of the considered cylinder. If the virtual boundary has not been violated, the extracted features are expected to pass through the virtual gauge.
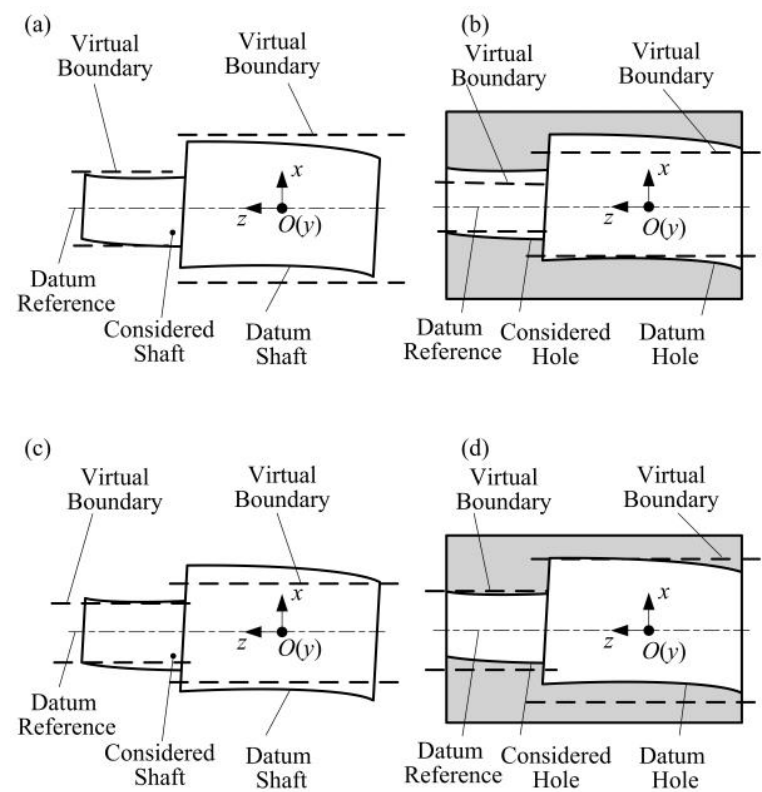

Figure.2. Virtual gauges for (a) a shaft with MMR, (b) a hole with MMR, (c) a shaft with LMR, and (d) a hole with LMR

In summary, there are five steps in the Coaxiality-DM evaluation method based on virtual gauge (Figure.3):

1. Preparation: Get EFC and EFD, and move them close to the origin and an axis of the coordinate system.

2. Size check: Check the size requirement by traditional methods.

3. Virtual gauge establishment: Calculate virtual boundary sizes, and establish the virtual gauge with coaxial cylinders for the considered cylinder and its datum cylinder.

4. Adjustment in the virtual gauge: Adjust the orientations and positions of the extracted features. In this paper, this step is described as a constrained optimization problem, and it is solved by an existing optimization method.

5. Conformity or nonconformity proof: The solution should be compared with the virtual boundary size of the considered cylinder. Conformity is proved only if both size and gauge checks conform to the five geometric requirements.

These steps are mathematically formulated in detail in Section.3.2. 


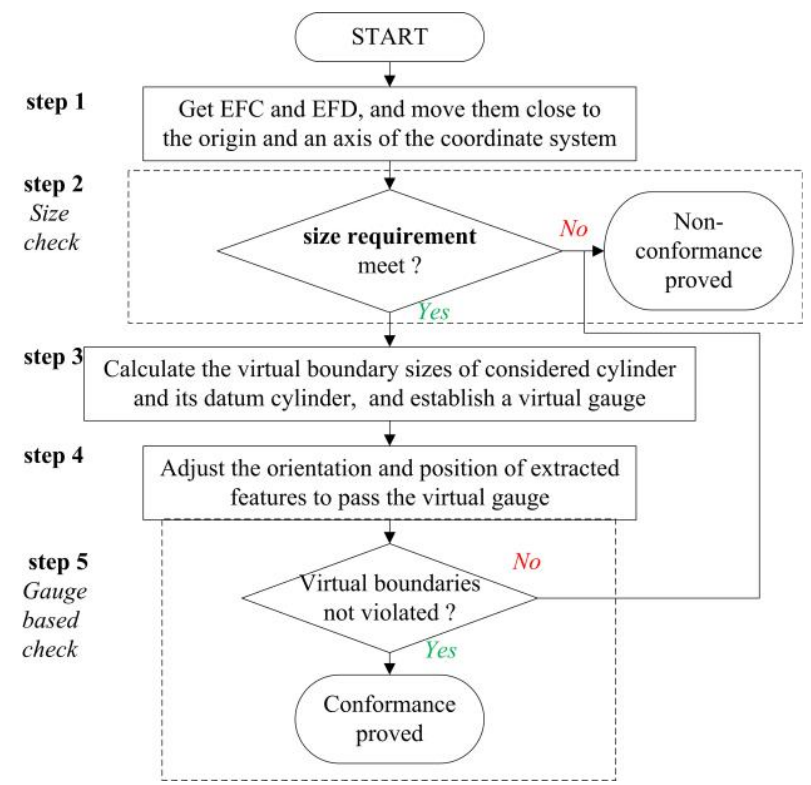

Figure.3. Verification of Coaxiality-DM

\subsection{Detailed steps of the evaluation}

\section{Step 1: Preparation}

EFD is obtained from an actual part as a set of coordinates $\left\{\boldsymbol{p}_{\mathrm{D}, \mathrm{ex}, n}\right\}$ of points $\left\{\boldsymbol{P}_{\mathrm{D}, \mathrm{ex}, n}\right\}$, and EFC is detected as a set of coordinates $\left\{\boldsymbol{p}_{\mathrm{C}, \mathrm{ex}, m}\right\}$ of points $\left\{\boldsymbol{P}_{\mathrm{C}, \mathrm{ex}, m}\right\}$, where $n=1,2, \ldots, N$, and $m=1,2, \ldots, M$ (Figure.4.(a)). In order to reduce the scale of point coordinates, which can result in a significant amplification of errors in numerical calculations, the extracted features are moved close to the origin and an axis of the evaluation coordinate system (the $z$-axis, in this paper). However, as an accurate movement is not necessary, least area, least squares, or some other rules can be applied to the movement. After the translations along the $x$-axis and $y$-axis (denoted as $\mathrm{d} x$ and $\mathrm{d} y$ ) and the rotations around the $x$-axis and the $y$-axis (denoted as $\mathrm{d} \alpha$ and $\mathrm{d} \beta$ ), the sets of points $\left\{\boldsymbol{P}_{\mathrm{D}, \mathrm{ex}, n}\right\}$ and $\left\{\boldsymbol{P}_{\mathrm{C}, \mathrm{ex}, m}\right\}$ are moved to $\left\{\boldsymbol{p}_{\mathrm{D}, \mathrm{int}, n}\right\}$ and $\left\{\boldsymbol{p}_{\mathrm{C}, \text { int }, m}\right\}$, respectively (Figure.4.(b)).

(a)

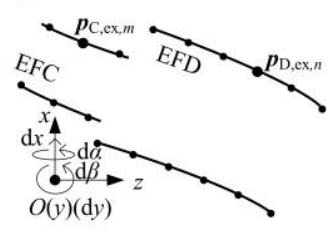

$\begin{array}{cc}\text { (c) Virtual } & \text { Virtual } \\ \text { Boundary } & \text { Boundary }\end{array}$

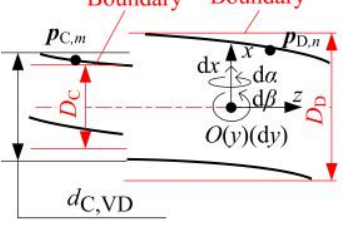

(e)

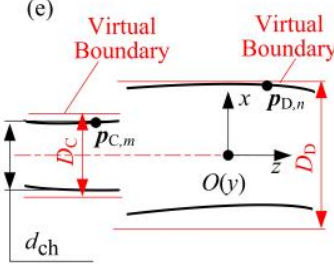

(b)

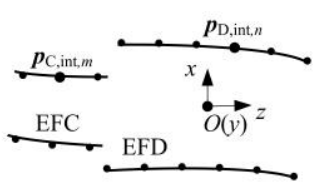

(d) Virtual Virtual
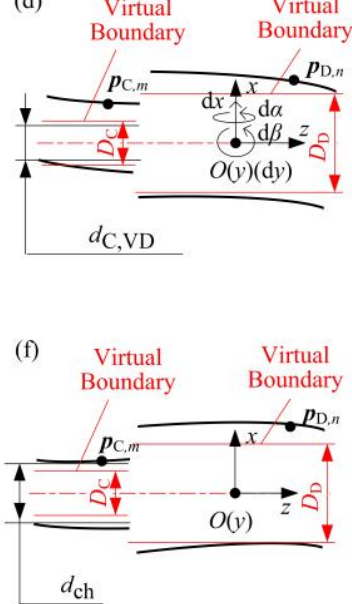

Figure.4. Extracted features (a) before the preparation, (b) after the preparation, (c) of shafts with MMR or holes with LMR in adjustment, (d) of holes with LMR or holes with MMR in adjustment, (e) of shafts with MMR or holes with LMR after adjustment, and (f) of holes with MMR or shafts with LMR after adjustment. 
According to the ISO standards $[17,18]$, the local size is defined as two-points-size, which is the diameter extension line of the least squares circle of a section and connects two points on the feature. However, it is difficult to find two scanned points to form an exact local size.

In contrast, the size of the maximum inscribed cylinder (MIC) is smaller than or equal to the minimum local size, and the size of the minimum circumscribed cylinder (MCC) is larger than or equal to the maximum local size. Consequently, an approximate approach is available to check the size by CMM measurements for a preliminary check: if the MCC of each cylinder is smaller than its maximum local size and the MIC of each cylinder is larger than its minimum local size, the size of the workpiece can be accepted. To evaluate an MIC or an MCC, a current optimization method, such as a geometric method or Quasi-Newton method, could be employed (Figure.5):

$$
\begin{aligned}
& \quad D_{\mathrm{Mm}}=2 C \min \left(C \max \left|\boldsymbol{p}_{i} \times \boldsymbol{n}_{z}\right|\right) \\
& \text { s.t. } \\
& \left\{\begin{array}{l}
\boldsymbol{p}_{i}=(\mathrm{d} x, \mathrm{~d} y, 0)+\boldsymbol{R}(\mathrm{d} \alpha, \mathrm{d} \beta, 0) \boldsymbol{p}_{\mathrm{CD}, \mathrm{int}, i}^{\mathrm{T}} \\
\mathrm{d} x, \mathrm{~d} y \in\left[-\tau_{\mathrm{M}}, \tau_{\mathrm{M}}\right] \\
\mathrm{d} \alpha, \mathrm{d} \beta \in\left[-\tau_{\mathrm{M}} / L, \tau_{\mathrm{M}} / L\right] \\
\tau_{\mathrm{M}}=\max \left|D_{\mathrm{CD}}-\right| \boldsymbol{p}_{\mathrm{CD}, \text { int }, i} \times \boldsymbol{n}_{z}|| \\
L=\left|\max \left(\boldsymbol{p}_{\mathrm{CD}, \text { in }, i} \cdot \boldsymbol{n}_{z}\right)-\min \left(\boldsymbol{p}_{\mathrm{CD}, \text { int }, i} \cdot \boldsymbol{n}_{z}\right)\right| \\
\boldsymbol{n}_{z}=(0,0,1)
\end{array}\right.
\end{aligned}
$$

where

$D_{\mathrm{Mm}}$ is the MIC or MCC of EFD or EFC;

$C$ is a factor which should be -1 for MIC and 1 for MCC;

$\left\{\boldsymbol{p}_{i}\right\}$ is the coordinates of points $\left\{\boldsymbol{P}_{\mathrm{C}, \text { int }, m}\right\}$ or $\left\{\boldsymbol{P}_{\mathrm{D}, \text { int }, n}\right\}$ after the translations $\mathrm{d} x$ and $\mathrm{d} y$ and the rotations $\mathrm{d} \alpha$ and $\mathrm{d} \beta$;

$i=1,2 \ldots N$ for EFD, while $i=1,2 \ldots M$ for EFC;

$\boldsymbol{R}(\mathrm{d} \alpha, \mathrm{d} \beta, 0)$ is a rotational matrix [8], which reflects the changes of coordinates when the extracted features rotate by $\mathrm{d} \alpha$ and $\mathrm{d} \beta$;

$\tau_{\mathrm{M}}$ is the greatest distance from the extracted feature to the virtual boundary;

$L$ is the length of EFD or EFC;

$D_{\mathrm{CD}}$ is the virtual boundary size of the considered feature or its datum cylinder;

$\boldsymbol{p}_{\mathrm{CD}, \text { int }, i}=\boldsymbol{p}_{\mathrm{D}, \text { int }, n}$ for EFD, while $\boldsymbol{p}_{\mathrm{CD}, \text { int }, i}=\boldsymbol{p}_{\mathrm{C}, \text { int }, m}$ for EFC; and

the limits of variables $\mathrm{d} x, \mathrm{~d} y, \mathrm{~d} \alpha$, and $\mathrm{d} \beta$ are provided to improve the efficiency and the stability of the optimization process.

(a)

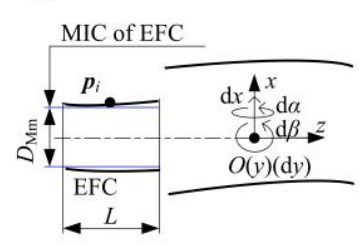

(c)

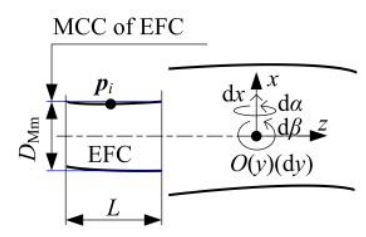

(b)

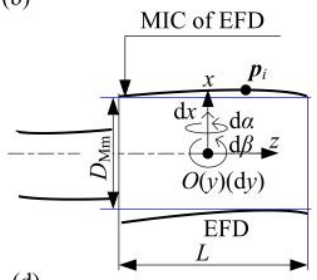

(d)

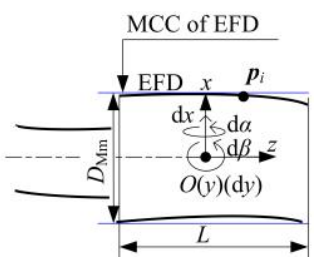

Figure.5. (a) MIC of EFC, (b) MIC of EFD, (c) MCC of EFC, and (d) MCC of EFD

\section{Step 3: Virtual gauge establishment}

When a material condition is applied to the datum cylinder, the datum cylinder is constrained by its virtual boundary. 
According to requirements II and V, the size of the virtual boundary is related to the tolerance specification instead of the actual part. Hence, when a part has less material than the specified material condition, the relative location and orientation between EFD and its virtual boundary are allowed to vary. Thus, it is difficult to derive a unique virtual boundary directly for and from EFD as well as a unique virtual boundary directly for and from EFC.

Therefore, a special method to generate the coaxial boundaries of the considered cylinder and its datum cylinder and construct a virtual gauge is necessary. In this paper, the relative motion between extracted features and their boundaries is described with a fixed virtual gauge and moving extracted features, where the common axis of the virtual gauge is fixed on an axis (the $z$-axis, in this paper, as shown in Figure.4.(c)-(d)).

According to requirement $\mathrm{V}$, the virtual boundary size of the datum cylinder $\left(D_{\mathrm{D}}\right)$ should be

$$
\left\{\begin{array}{l}
\text { requirement }(\text { V. a })\left\{\begin{array}{l}
\text { For MMR: } D_{\mathrm{D}}=D_{\mathrm{M}, \mathrm{D}} \\
\text { For LMR }: D_{\mathrm{D}}=D_{\mathrm{L}, \mathrm{D}}
\end{array}\right. \\
\text { requirement }(\mathbf{V} . \mathbf{b})\left\{\begin{array}{l}
\text { For Shaft and } M M R: D_{\mathrm{D}}=D_{\mathrm{M}, \mathrm{D}}+t_{\mathrm{D}} \\
\text { For Hole and } M M R: D_{\mathrm{D}}=D_{\mathrm{M}, \mathrm{D}}-t_{\mathrm{D}} \\
\text { For Hole and } L M R: D_{\mathrm{D}}=D_{\mathrm{L}, \mathrm{D}}+t_{\mathrm{D}} \\
\text { For Shaft and } L M R: D_{\mathrm{D}}=D_{\mathrm{L}, \mathrm{D}}-t_{\mathrm{D}}
\end{array}\right.
\end{array}\right.
$$

where $t_{\mathrm{D}}$ is the coaxiality tolerance of a datum feature; and $D_{\mathrm{M}, \mathrm{D}}$ and $D_{\mathrm{L}, \mathrm{D}}$ are the MMS and LMS of the datum feature, respectively.

According to requirement IV, the virtual boundary size of the considered cylinder $\left(D_{\mathrm{C}}\right)$ should be

$$
\left\{\begin{array}{l}
\text { For Shaft and MMR: } D_{\mathrm{C}}=D_{\mathrm{M}, \mathrm{C}}+t_{\mathrm{C}} \\
\text { For Hole and MMR: } D_{\mathrm{C}}=D_{\mathrm{M}, \mathrm{C}}-t_{\mathrm{C}} \\
\text { For Hole and LMR: } D_{\mathrm{C}}=D_{\mathrm{L}, \mathrm{C}}+t_{\mathrm{C}} \\
\text { For Shaft and LMR: } D_{\mathrm{C}}=D_{\mathrm{L}, \mathrm{C}}-t_{\mathrm{C}}
\end{array}\right.
$$

where $t_{\mathrm{C}}$ is the coaxiality tolerance of a considered feature; and $D_{\mathrm{M}, \mathrm{C}}$ and $D_{\mathrm{L}, \mathrm{C}}$ are the MMS and LMS of the considered feature, respectively.

\section{Step 4: Adjustment in the virtual gauge}

When a real part with an MMR Coaxiality-DM tolerance is verified by a real gauge, the real part is adjusted by translating and rotating it to try to pass it through the real gauge. Similarly, when the extracted features are verified by a virtual gauge, they should be adjusted to try to pass them through the virtual gauge. In the adjustment process, EFD should not violate its virtual boundary, while EFC should be as coaxial as possible with its virtual boundary.

Consequently, the MMVB-coaxial envelope of EFC $\left(d_{\mathrm{C}, \mathrm{VD}}\right)$ should be minimized (for a shaft with MMR or a hole with LMR) or maximized (for a shaft with LMR or a hole with MMR), and the MMVB-coaxial envelope should be coaxial with the virtual boundaries of the considered cylinder and its datum cylinder (Figure.4.(c)-(f)). Thus, the minimum MMVB-coaxial envelope of EFC $\left(d_{\mathrm{ch}}\right)$ can be obtained by the adjustment described in Formula (4) and solved by a current optimization method such as SQP.

$$
\begin{aligned}
& d_{\mathrm{ch}}=C_{\mathrm{C}} \min d_{\mathrm{C}, \mathrm{VD}} \\
& d_{\mathrm{C}, \mathrm{VD}}=2 \max \left(C_{\mathrm{C}}\left|\boldsymbol{p}_{\mathrm{C}, m} \times \boldsymbol{n}_{z}\right|\right) \\
& \qquad \begin{array}{l}
\boldsymbol{p}_{\mathrm{C}, m}=(\mathrm{d} x, \mathrm{~d} y, 0)+\boldsymbol{R}(\mathrm{d} \alpha, \mathrm{d} \beta, 0) \boldsymbol{p}_{\mathrm{C}, \mathrm{int}, m}^{\mathrm{T}} \\
\boldsymbol{p}_{\mathrm{D}, n}=(\mathrm{d} x, \mathrm{~d} y, 0)+\boldsymbol{R}(\mathrm{d} \alpha, \mathrm{d} \beta, 0) \boldsymbol{p}_{\mathrm{D}, \mathrm{int}, n}^{\mathrm{T}} \\
2 C_{\mathrm{D}}\left|\boldsymbol{p}_{\mathrm{D}, n} \times \boldsymbol{n}_{z}\right| \leq C_{\mathrm{D}} D_{\mathrm{D}} \\
\mathrm{d} x, \mathrm{~d} y \in\left[-\tau_{M}, \tau_{M}\right] \\
\mathrm{d} \alpha, \mathrm{d} \beta \in\left[-\tau_{M} / L_{\mathrm{D}}, \tau_{M} / L_{\mathrm{D}}\right] \\
\tau_{\mathrm{M}}=\max \left|D_{\mathrm{D}}-\right| \boldsymbol{p}_{\mathrm{D}, \text { int }, n} \times \boldsymbol{n}_{z}|| \\
L_{\mathrm{D}}=\left|\max \left(\boldsymbol{p}_{\mathrm{D}, \text { int }, n} \cdot \boldsymbol{n}_{z}\right)-\min \left(\boldsymbol{p}_{\mathrm{D}, \text { int }, n} \cdot \boldsymbol{n}_{z}\right)\right| \\
\boldsymbol{n}_{z}=(0,0,1) \\
m=1,2, \cdots, M ; n=1,2, \cdots, N
\end{array}
\end{aligned}
$$


where $C_{\mathrm{C}}$ and $C_{\mathrm{D}}$ are the factors for the considered cylinder and its datum cylinder, respectively; the values of $C_{\mathrm{C}}$ and $C_{\mathrm{D}}$ should be 1 for shafts with MMR or holes with LMR, whereas $C_{\mathrm{C}}$ and $C_{\mathrm{D}}$ should be -1 for shafts with LMR or holes with MMR. Additionally, $\boldsymbol{p}_{\mathrm{C}, m}$ and $\boldsymbol{p}_{\mathrm{D}, n}$ are the coordinates of points $\left\{\boldsymbol{P}_{\mathrm{C}, \text { int }, m}\right\}$ and $\left\{\boldsymbol{P}_{\mathrm{D}, \text { int }, n}\right\}$ after the translations $\mathrm{d} x$ and $\mathrm{d} y$ and the rotations $\mathrm{d} \alpha$ and $\mathrm{d} \beta$, respectively, and $L_{\mathrm{D}}$ is the measured length of the datum feature. The limits of variables $\mathrm{d} x, \mathrm{~d} y, \mathrm{~d} \alpha$, and $\mathrm{d} \beta$ are also provided to improve the efficiency and the stability of the optimization process.

\section{Step 5: Conformity or nonconformity proof}

After the adjustment of extracted features, if the MMVB-coaxial envelope of EFC does not violate its virtual boundary, the extracted features can pass through the virtual gauge (Figure.4.(e)-(f)). This means requirements II-V are satisfied and can be formulated as follows.

comforming features $\Leftrightarrow$

$$
\left\{\begin{array}{c}
\text { SHAFT with MMR or HOLE with LMR: } \\
\quad d_{\mathrm{ch}} \leq D_{\mathrm{C}} \\
\text { SHAFT with LMR or HOLE with MMR: } \\
d_{\mathrm{ch}} \geq D_{\mathrm{C}}
\end{array}\right.
$$

If requirement $\mathrm{I}$ is satisfied in step 2 and requirements II-V are satisfied in step 5, conformity is proved; otherwise, nonconformity is detected.

\section{Case study}

\subsection{Illustration for the proposed method}

The MMR Coaxiality-DM tolerance for a stepped shaft is specified in Figure.6, and the values of EFC and EFD (measured by a CMM) for a real part are presented in Figure.7. The result was utilized to demonstrate whether the actual part conformed to the MMR Coaxiality-DM tolerance.

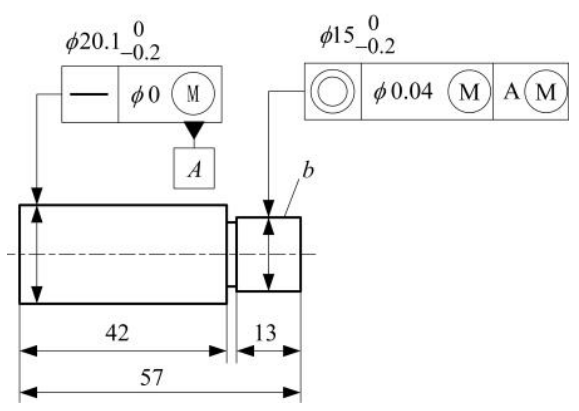

Figure.6. Stepped shaft with the MMR Coaxiality-DM tolerance

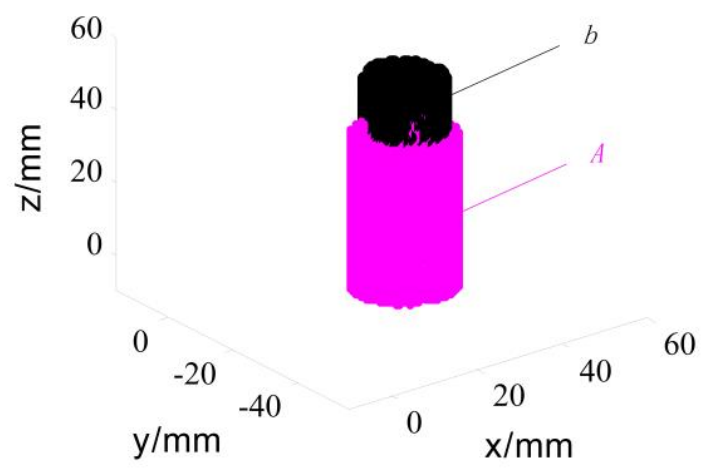

Figure.7. Extracted features from measurements

The CMM used in the study was the GLOBAL CLASSIC SR 07.10.07 produced by Hexagon Metrology (Qing Dao, China). The uncertainty of the non-contact measurement by this machine was $0.02 \mathrm{~mm}$. The part was non-contact scanned from three directions, which were each described in terms of angle A (around the $x$-axis) and angle B (around the $z$-axis) 
in that order as follows: $60^{\circ}$ and $0^{\circ}$; $-60^{\circ}$ and $60^{\circ}$; and $-60^{\circ}$ and $-60^{\circ}$. Meanwhile, the interval between scanning lines was $2 \mathrm{~mm}$, and the interval between points on the scanning lines was the same.

\section{Step 1: Preparation}

The EFD of datum cylinder $A$ and the EFC of considered cylinder $b$ were obtained from the point sets $\left\{\boldsymbol{P}_{\mathrm{D}, \mathrm{ex}, n}\right\}$ and $\left\{\boldsymbol{P}_{\mathrm{C}, \mathrm{ex}, m}\right\}$, respectively (Figure.7). The coordinates of $\left\{\boldsymbol{P}_{\mathrm{D}, \mathrm{ex}, n}\right\}$ in the set $\left\{\boldsymbol{p}_{\mathrm{D}, \mathrm{ex}, n}\right\}$ were $\left\{\left(x_{\mathrm{D}, \mathrm{ex}, n}, y_{\mathrm{D}, \mathrm{ex}, n}, z_{\mathrm{D}, \mathrm{ex}, n}\right)^{\mathrm{T}}\right\}$, whereas the coordinates of $\left\{\boldsymbol{P}_{\mathrm{C}, \mathrm{ex}, m}\right\}$ in the set $\left\{\boldsymbol{p}_{\mathrm{C}, \mathrm{ex}, m}\right\}$ were $\left\{\left(x_{\mathrm{C}, \mathrm{ex}, m}, y_{\mathrm{C}, \mathrm{ex}, m}, z_{\mathrm{C}, \mathrm{ex}, m}\right)^{\mathrm{T}}\right\}$, where $n=1,2, \ldots, 2093$ and $m=1,2, \ldots, 448$.

Then, the extracted features were moved close to the origin and the z-axis. In our study, the stepped shaft was roughly parallel to the $z$-axis due to its orientation during the measurement. Therefore, in order to translate the extracted features to the origin, the average of $\boldsymbol{p}_{\mathrm{D}, \mathrm{ex}, n}$ was set as zero. Hence, the sets $\left\{\boldsymbol{p}_{\mathrm{D}, \mathrm{ex}, n}\right\}$ and $\left\{\boldsymbol{p}_{\mathrm{C}, \mathrm{ex}, m}\right\}$ were changed to $\left\{\boldsymbol{p}_{\mathrm{D}, \text { int }, n}\right\}$ and $\left\{\boldsymbol{p}_{b, \text { int }, m}\right\}$, respectively.

$$
\begin{gathered}
\boldsymbol{p}_{m}=\frac{1}{2093} \sum_{i=1}^{2093} \boldsymbol{p}_{\mathrm{D}, \mathrm{ex}, n} \\
\left\{\begin{array}{l}
\boldsymbol{p}_{\mathrm{C}, \mathrm{ini}, m}=\boldsymbol{p}_{\mathrm{C}, \mathrm{ex}, m}-\boldsymbol{p}_{m} \\
\boldsymbol{p}_{\mathrm{D}, \mathrm{ini}, n}=\boldsymbol{p}_{\mathrm{D}, \mathrm{ex}, n}-\boldsymbol{p}_{m}
\end{array}\right.
\end{gathered}
$$

\section{Step 2: Size check}

To evaluate MCC and MIC, the SQP function was employed in MATLAB R2013a. Then the value of $D_{\mathrm{Mm}}$ was calculated by Formula (1): the sizes of MCC and MIC for the EFD of datum cylinder $A$ were $20.091 \mathrm{~mm}$ and $19.915 \mathrm{~mm}$, respectively, whereas the sizes of MCC and MIC for the EFC of considered cylinder $b$ were $14.996 \mathrm{~mm}$ and $14.863 \mathrm{~mm}$, respectively. According to the size tolerances in Figure.6 (20.1 mm to $19.9 \mathrm{~mm}$ for feature $A$ and $15.0 \mathrm{~mm}$ to $14.8 \mathrm{~mm}$ for feature $b$ ), the actual sizes of feature $A$ and feature $b$ met size requirement $\mathrm{I}$.

\section{Step 3: Virtual gauge establishment}

A virtual gauge was established, which consisted of two coaxial holes: the MMVB of considered cylinder $b$ and the MMVB of its datum cylinder $A$. In this case, the common axis of the two MMVBs was on the $z$-axis. As specified in Figure.6, the datum cylinder $A$ was a shaft with a form tolerance and MMR. Thus, according to requirement $\mathrm{V}(\mathrm{b})$, the MMVB size of the datum cylinder $\left(D_{\mathrm{D}}\right)$ was calculated by Formula (2):

$$
D_{\mathrm{D}}=D_{\mathrm{M}, \mathrm{D}}+t_{\mathrm{D}}=20.1+0=20.1
$$

Since the considered cylinder $b$ was a shaft, the MMVB size of the considered cylinder $\left(D_{\mathrm{C}}\right)$ was calculated by Formula (3) to meet requirement IV:

$$
D_{\mathrm{C}}=D_{\mathrm{M}, \mathrm{C}}+t_{\mathrm{C}}=15+0.04=15.04
$$

where $D_{\mathrm{M}, \mathrm{C}}$ and $D_{\mathrm{M}, \mathrm{D}}$ are the MMS of considered cylinder $b$ and its datum cylinder $A$, respectively; $t_{\mathrm{D}}$ is the straightness tolerance of datum cylinder $A$; and $t_{\mathrm{C}}$ is the coaxiality tolerance of considered cylinder $b$.

\section{Step 4: Adjustment in the virtual gauge}

The minimum MMVB-coaxial envelope of EFC $\left(d_{\mathrm{ch}}\right)$ was calculated by Formula (4):

$$
\begin{aligned}
& d_{\mathrm{ch}}=\min d_{\mathrm{C}, \mathrm{VD}}, \quad d_{\mathrm{C}, \mathrm{VD}}=2 \max \left|\boldsymbol{p}_{\mathrm{C}, m} \times \boldsymbol{n}_{z}\right| \\
& \text { s.t. }
\end{aligned}
$$




$$
\left\{\begin{array}{l}
\boldsymbol{p}_{\mathrm{C}, m}=(\mathrm{d} x, \mathrm{~d} y, 0)+\boldsymbol{R}(\mathrm{d} \alpha, \mathrm{d} \beta, 0) \boldsymbol{p}_{\mathrm{C}, \mathrm{Tini}, m}^{\mathrm{T}} \\
\boldsymbol{p}_{\mathrm{D}, n}=(\mathrm{d} x, \mathrm{~d} y, 0)+\boldsymbol{R}(\mathrm{d} \alpha, \mathrm{d} \beta, 0) \boldsymbol{p}_{\mathrm{D}, \mathrm{ini}, n}^{\mathrm{T}} \\
2\left|\boldsymbol{p}_{\mathrm{D}, n} \times \boldsymbol{n}_{z}\right| \leq D_{\mathrm{D}}=20.1 \\
\mathrm{~d} x, \mathrm{~d} y \in\left[-\tau_{\mathrm{M}}, \tau_{\mathrm{M}}\right] \\
\mathrm{d} \alpha, \mathrm{d} \beta \in\left[-\tau_{\mathrm{M}} / L_{\mathrm{D}}, \tau_{\mathrm{M}} / L_{\mathrm{D}}\right]=\left[-\tau_{\mathrm{M}} / 41.224, \tau_{\mathrm{M}} / 41.224\right] \quad(9) \\
\tau_{\mathrm{M}}=\max \left|D_{\mathrm{D}}-\right| \boldsymbol{p}_{\mathrm{D}, \mathrm{nin}, n} \times \boldsymbol{n}_{z}|| \\
L_{\mathrm{D}}=\left|\max \left(\boldsymbol{p}_{\mathrm{D}, \mathrm{nin}, n} \cdot \boldsymbol{n}_{z}\right)-\min \left(\boldsymbol{p}_{\mathrm{D}, \mathrm{ini}, n} \cdot \boldsymbol{n}_{z}\right)\right| \\
\boldsymbol{n}_{z}=(0,0,1) \\
n=1,2, \cdots, 2093 ; m=1,2, \cdots, 448
\end{array}\right.
$$

where $\boldsymbol{p}_{\mathrm{D}, n}$ and $\boldsymbol{p}_{\mathrm{C}, m}$ are the coordinates of EFD and EFC after adjustment; and the extracted features translate by $\mathrm{d} x$ and $\mathrm{d} y$ and rotate by $\mathrm{d} \alpha$ and $\mathrm{d} \beta$ during the adjustment.

The optimization problem was solved by several functions in MATLAB R2013a, and $d_{\text {ch }}$ was calculated to be 15.006 $\mathrm{mm}$ by the inner point method in 2.222 seconds, $15.007 \mathrm{~mm}$ by ASM in 3.915 seconds, and $15.006 \mathrm{~mm}$ by SQP in 267.459 seconds.

\section{Step 5: Conformity or nonconformity proof}

The geometric error represented by $d_{\text {ch }}$ was checked by Formula (5):

$d_{\mathrm{ch}}=15.006<15.04=D_{\mathrm{C}}$

$\Rightarrow$ conforming features

\subsection{Results}

The uncertainty of the non-contact measurement by this machine was $0.02 \mathrm{~mm}$, which was less than $60 \%$ of the difference between $D_{\mathrm{C}}$ and $d_{\mathrm{ch}}(15.04-15.006=0.034 \mathrm{~mm})$; therefore, the result could be accepted. Hence, both the considered cylinder of the workpiece and its datum cylinder were proved to conform to the MMR Coaxiality-DM tolerance specified in Figure.6.

\section{Comparison and discussion}

In order to analyze the accuracy of the proposed method, the T-Map method was employed to evaluate the part in Section.4, and the results were compared with the results of the proposed method. As shown in Figure.8.(a), The T-Map method was used to transfer the MMR Coaxiality-DM tolerance of the stepped shaft in Section.4 to a datum fixed coaxiality with the following steps [14]:

1. The MCC of datum shaft $A$ was obtained (20.091 mm) in Section.4.2;

2. The size tolerance of considered datum shaft $b$ was kept the same (from $15+0 \mathrm{~mm}$ to $15-0.2 \mathrm{~mm}$ );

3. A new coaxiality tolerance $(0.049 \mathrm{~mm})$ was generated by a Boolean operation depending on the envelope size of datum feature $A$, the size tolerance of considered shaft $b$, and the original coaxiality $(0.04 \mathrm{~mm})$;

4. MMR was only kept for the considered shaft; and

5. An EFD-coaxial virtual boundary was obtained $(15.049 \mathrm{~mm})$.

For verification, the EFD-coaxial envelope size of the considered shaft $b\left(d_{\mathrm{C}, \mathrm{T}}\right.$, as shown in Figure.8.(c)) could be evaluated by Formula (11):

$$
d_{\mathrm{C}, \mathrm{T}}=2 \max \left|\boldsymbol{p}_{\mathrm{C}, \mathrm{T}, \mathrm{m}} \times \boldsymbol{n}_{z}\right|
$$

where $\boldsymbol{p}_{\mathrm{C}, \mathrm{T}, m}$ is the coordinates of points $\left\{\boldsymbol{P}_{\mathrm{C}, \mathrm{int}, m}\right\}$ after the translations $\mathrm{d} x_{\mathrm{T}}$ and $\mathrm{d} y_{\mathrm{T}}$ and the rotations $\mathrm{d} \alpha_{\mathrm{T}}$ and $\mathrm{d} \beta_{\mathrm{T}}$; $\boldsymbol{p}_{\mathrm{C}, \mathrm{T}, m}=\left(\mathrm{d} x_{\mathrm{T}}, \mathrm{d} y_{\mathrm{T}}, 0\right)+\boldsymbol{R}\left(\mathrm{d} \alpha_{\mathrm{T}}, \mathrm{d} \beta_{\mathrm{T}}, 0\right) \boldsymbol{p}_{\mathrm{C}, \mathrm{ini}, m}^{\mathrm{T}}$, as shown in Figure.8.(b) and (c); $m=1,2, \cdots, 448 ;$ and $\mathrm{d} x_{\mathrm{T}}, \mathrm{d} y_{\mathrm{T}}, \mathrm{d} \alpha_{\mathrm{T}}$, and $\mathrm{d} \beta_{\mathrm{T}}$ indicate the location and orientation of the envelope of EFD (fixed-datum $A$ ) and constitute the solution of the constrained 
optimization Formula (12), as shown in Figure.8.(b) and (c).

$$
d_{\mathrm{D}, \mathrm{T}}=2 \min \left(\max \left|\boldsymbol{p}_{\mathrm{D}, \mathrm{T}, n} \times \boldsymbol{n}_{z}\right|\right)
$$

s.t.

$$
\left\{\begin{array}{l}
\boldsymbol{p}_{\mathrm{D}, n}=(\mathrm{d} x, \mathrm{~d} y, 0)+\boldsymbol{R}(\mathrm{d} \alpha, \mathrm{d} \beta, 0) \boldsymbol{p}_{\mathrm{D}, \mathrm{ini}, n}^{T} \\
\boldsymbol{n}_{z}=(0,0,1) \\
m=1,2, \cdots, 2093
\end{array}\right.
$$

where $\boldsymbol{p}_{\mathrm{D}, \mathrm{T}, n}$ represents the coordinates of points $\left\{\boldsymbol{P}_{\mathrm{D}, \mathrm{int}, n}\right\}$ after the translations $\mathrm{d} x$ and $\mathrm{d} y$ and the rotations $\mathrm{d} \alpha$ and $\mathrm{d} \beta$; and $d_{\mathrm{D}, \mathrm{T}}$ is the envelope size of the datum shaft $A$.

The EFD-coaxial envelope size of the considered shaft $b$ was solved $(15.052 \mathrm{~mm}$ ) in MATLAB R2013a in 0.034 seconds. Since the EFD-coaxial envelope size was greater than the EFD-coaxial virtual boundary $(15.049 \mathrm{~mm})$, the conformity to the original MMR Coaxiality-DM specification was not proved by the T-Map method. In contrast, the conformance was proved by the proposed method. Additionally, as the difference between $D_{\mathrm{C}}$ and $d_{\mathrm{ch}}(0.034 \mathrm{~mm})$ was $85 \%$ of the coaxiality tolerance $(0.04 \mathrm{~mm})$, the error was far from its limit.

(a)

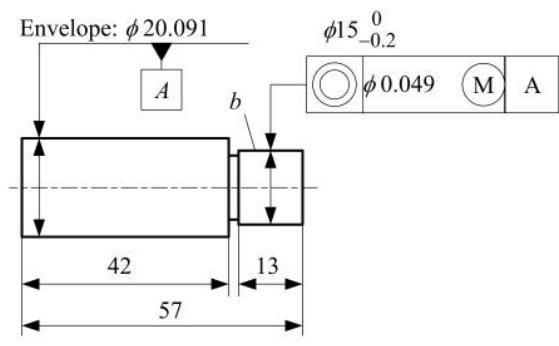

(b)

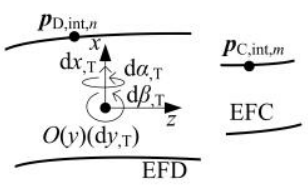

(c)

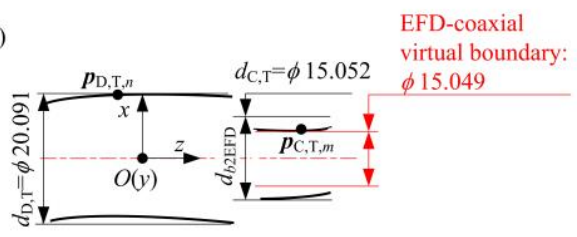

Figure.8. Transformed specification and verification by T-Map method

The T-Map method has more stringent requirements than the proposed method. This is because the proposed method exactly describes all five requirements of the MMR Coaxiality-DM tolerance to prove conformance or nonconformance, whereas the T-Map method transfers the MMR Coaxiality-DM tolerance to a stricter traditional tolerance to ensure a higher eligibility-rate production. The proposed evaluation method is less expensive, but it requires much more time (more than 2.222 seconds) than the T-Map method (0.034 seconds).

\section{Conclusion}

The evaluation method for Coaxiality-DM tolerance is a key research area for computer-aided tolerance design. All five requirements of Coaxiality-DM tolerance were introduced, and the geometries of a real gauge and the movement of a real part were analyzed. In order to avoid the limits of real gauges (such as high cost and unavailable LMR cases), an evaluation method based on a virtual gauge was proposed. In the proposed method, the virtual gauge is composed of two coaxial virtual cylinders, and the real part is represented by extracted features. After that, the extracted features are adjusted in the virtual gauge to try to pass them through the virtual gauge. The process is realized by five steps and five formulas.

In addition, the evaluation of a stepped shaft with an MMR Coaxiality-DM tolerance was presented for a case study. 
In the case study, the minimum MMVB-coaxial envelope of the considered shaft $\left(d_{\mathrm{ch}}\right)$ was evaluated in 2.222 seconds, and the result was $d_{\mathrm{ch}}=15.006 \mathrm{~mm}$. As $d_{\mathrm{ch}}$ was less than the MMVS of the datum shaft $(15.04 \mathrm{~mm})$, the considered shaft and its datum shaft were proved to be able to pass through the virtual gauge.

Finally, evaluation according to the T-Map method was presented for comparative study. The MMR Coaxiality-DM tolerance was transferred to an MMR coaxiality tolerance, and the EFD-coaxial envelope of the considered shaft was obtained $\left(d_{\mathrm{C}, \mathrm{T}}=15.052 \mathrm{~mm}\right)$ in 0.034 seconds. As $d_{\mathrm{C}, \mathrm{T}}$ was greater than the transferred EFD-coaxial virtual boundary of the considered shaft $(15.04 \mathrm{~mm})$, the result did not suggest accepting the part. From the comparison, it was found that the proposed method has greater accuracy, while the T-Map method has stricter requirements. However, the proposed method requires more time.

According to the case study, even when SQP or any other optimization technique is introduced for a medium-scale or large-scale constrained problem, the proposed method takes more time than an approximation method such as the T-Map method. In contrast, compared with traditional optimization techniques, geometric search methods usually take less time in error evaluation [6]. Therefore, in the future, our team is going to develop a geometric algorithm based on the virtual gauge for Coaxiality-DM evaluation.

\section{Acknowledgments}

This work was supported by the National Natural Science Foundation of China [51765012]; Guangxi Key Laboratory of Manufacturing Systems and Advanced Manufacturing Technology [16-380-12-009Z]; and the Innovation Project of Guangxi Graduate Education [YCBZ2017049].

\section{Nomenclature}

C

$C_{\mathrm{C}}$

$C_{\mathrm{D}}$

$D_{\mathrm{C}}$

$D_{\mathrm{CD}}$

$D_{\mathrm{D}}$

$D_{\mathrm{L}, \mathrm{C}}$

$D_{\mathrm{L}, \mathrm{D}}$

$D_{\mathrm{M}, \mathrm{C}}$

$D_{\mathrm{M}, \mathrm{D}}$

$D_{\mathrm{Mm}}$

$\mathrm{d} \alpha$

$\mathrm{d} \alpha, \mathrm{T}$

$\mathrm{d} \beta$

$\mathrm{d} \beta \beta_{\text {, }}$

$d_{\mathrm{ch}}$

$d_{\mathrm{C}, \mathrm{T}}$

$d_{\mathrm{C}, \mathrm{VD}}$

$d_{\mathrm{D}, \mathrm{T}}$

$\mathrm{d} x$

$\mathrm{d} x_{, \mathrm{T}}$

$\mathrm{d} y$

$\mathrm{d} y_{,} \mathrm{T}$ $i$

$L$

A factor which should be -1 for MIC and 1 for MCC (Figure.5)

Factor for the considered cylinder

Factor for the datum cylinder

The virtual boundary size of the considered cylinder (Figure.4)

$D_{\mathrm{C}}$ for the considered feature or $D_{\mathrm{D}}$ for its datum cylinder

The virtual boundary size of the datum cylinder (Figure.4)

The LMS of the considered feature

The LMS of the datum feature

The MMS of the considered feature

The MMS of the datum feature

The size of the maximum inscribed cylinder or the minimum circumscribed cylinder of EFD or EFC

Rotations around the $x$-axis (Figure.4)

Rotations around the $x$-axis of the movement in T-Map method (Figure.8)

Rotations around the $y$-axis (Figure.4)

Rotations around the $y$-axis of the movement in T-Map method (Figure.8)

The minimum MMVB-coaxial envelope of EFC (Figure.4)

The EFD-coaxial envelope size of the considered shaft $b$ (Figure.8)

The MMVB-coaxial envelope of EFC (Figure.4)

The envelope size of the datum shaft $A$ (Figure.8)

Translations along the $x$-axis (Figure.4)

Translations along the $x$-axis of the movement in T-Map method (Figure.8)

Translations along the $y$-axis (Figure.4)

Translations along the $y$-axis of the movement in T-Map method (Figure.8)

$n$ for EFD or $m$ for EFC

The length of EFD or EFC (Figure.5)

The length of EFD

The amount of points of EFC 


\begin{tabular}{|c|c|}
\hline$m$ & The identifier number of points of EFC \\
\hline$N$ & The amount of points of EFD \\
\hline$n$ & The identifier number of points of EFD \\
\hline $\boldsymbol{n}_{z}$ & The unit vector along the $z$-axis \\
\hline $\boldsymbol{P}_{\mathrm{C}, \mathrm{ex}, m}$ & The $m$-th point on an actual toleranced cylinder \\
\hline $\boldsymbol{P}_{\mathrm{D}, \mathrm{ex}, n}$ & The $n$-th point on an actual datum cylinder \\
\hline $\boldsymbol{p}_{\mathrm{CD}, \mathrm{int}, i}$ & $\boldsymbol{p}_{\mathrm{D}, \mathrm{int}, n}$ for EFD, while $\boldsymbol{p}_{\mathrm{CD}, \text { int }, i}=\boldsymbol{p}_{\mathrm{C}, \text { int }, m}$ for EFC \\
\hline $\boldsymbol{p}_{\mathrm{C}, \mathrm{ex}, m}$ & The original coordinates of $\boldsymbol{P}_{\mathrm{C}, \mathrm{ex}, m}($ Figure.4) \\
\hline $\boldsymbol{p}_{\mathrm{C}, \mathrm{int}, m}$ & The coordinates of $\boldsymbol{P}_{\mathrm{C}, \mathrm{ex}, m}$ after preparation (Figure.4) \\
\hline $\boldsymbol{p}_{\mathrm{C}, m}$ & The coordinates of $\boldsymbol{P}_{\mathrm{C}, \mathrm{ex}, m}$ after final adjustment (Figure.4) \\
\hline$p_{\mathrm{C}, \mathrm{T}, m}$ & The coordinates of $\boldsymbol{P}_{\mathrm{C}, \text { int }, m}$ after the movement in T-Map method (Figure.8) \\
\hline$p_{\mathrm{D}, \mathrm{ex}, n}$ & The original coordinates of $\boldsymbol{P}_{\mathrm{D}, \mathrm{ex}, n}($ Figure.4) \\
\hline$p_{\mathrm{D}, \mathrm{int}, n}$ & The coordinates of $\boldsymbol{P}_{\mathrm{D}, \mathrm{ex}, n}$ after preparation (Figure.4) \\
\hline $\boldsymbol{p}_{\mathrm{D}, n}$ & The coordinates of $\boldsymbol{P}_{\mathrm{D}, \mathrm{ex}, n}$ after final adjustment (Figure.4) \\
\hline $\boldsymbol{p}_{\mathrm{D}, \mathrm{T}, n}$ & The coordinates of $\boldsymbol{P}_{\mathrm{D}, \mathrm{ex}, n}$ after the movement in T-Map method (Figure.8) \\
\hline $\boldsymbol{p}_{i}$ & The coordinates of $\boldsymbol{P}_{\mathrm{C}, \text { int }, m}$ or $\boldsymbol{P}_{\mathrm{D}, \text { int }, n}$ after movement, in size checking (Figure.5) \\
\hline $\boldsymbol{R}(\mathrm{d} \alpha, \mathrm{d} \beta, 0)$ & A rotational matrix [8], reflecting the changes of coordinates of a point after rotating by $\mathrm{d} \alpha$ and $\mathrm{d} \beta$ \\
\hline$t_{\mathrm{C}}$ & The coaxiality tolerance of datum feature \\
\hline$t_{\mathrm{D}}$ & The form tolerance of datum feature \\
\hline $\mathrm{M}$ & The greatest distance from the extracted feature to the virtual boundary \\
\hline
\end{tabular}

\section{References}

[1]ISO 2692:2014, Geometrical product specifications (GPS) -Geometrical tolerancing- Maximum material requirement (MMR), least material requirement (LMR) and reciprocity requirement (RPR).

[2]B. Anselmetti, L. Pierre. Complementary writing of maximum and least material requirements, with an extension to complex surfaces, Procedia CIRP, 43 (2016) 220-225.

[3]F. Liu, G.H. Xu, L. Lin, et al. Least squares evaluations for form and profile errors of ellipse using coordinate data, Chin. J. Mach. Eng. 29 (2016) 1-9.

[4]Z.M. Cao, Y. Wu, J. Han. Roundness deviation evaluation method based on statistical analysis of local least square circles, Meas. Sci. Technol. 28 (2017) 105017.

[5]P. Li, X.M. Ding, J.B. Tan, et al. A hybrid method based on reduced constraint region and convex-hull edge for flatness error evaluation, Precis. Eng. 45 (2016) 168-175.

[6]R. Calvo, G. Emilio, R. Domingo. Vectorial method of minimum zone tolerance for flatness, straightness, and their uncertainty estimation, Int. J. Precis. Eng. Manuf. 15 (2014) 31-44.

[7]F. Liu, G.H. Xu, L. Lin, et al. Minimum zone evaluation of sphericity deviation based on the intersecting chord method in Cartesian coordinate system, Precis. Eng. 45 (2016) 216-229.

[8]V. Radlovački, M. Hadžistević, B. Štrbac, et al. Evaluating minimum zone flatness error using new method—Bundle of plains through one point, Precis. Eng. 43 (2016) 554-562.

[9]P. Zheng, J.Q. Wu, L.N. Zhang. Research of the on-line evaluating the cylindricity error technology based on the new generation of GPS, Procedia Eng. 174 (2017) 402-409.

[10]V.K. Pathak, S. Kumar, C. Nayak, et al. Evaluating geometric characteristics of planar surfaces using improved particle swarm optimization, Meas. Sci. Rev. 17 (2017) 187-196.

[11]L. Jin, Y.P. Chen, H.Y. Lu, et al. Roundness error evaluation based on differential evolution algorithm, Appl. Mech. Mater. 670-671 (2014) 1285-1289.

[12]A.L. Lang, Z.J. Song, G.Y. He, et al. Profile error evaluation of free-form surface using sequential quadratic programming algorithm, Precis. Eng. 47 (2017) 344-352.

[13]M. Giordano, S. Samper, J.P. Petit. Tolerance analysis and synthesis by means of deviation domains, axis-symmetric cases, in: 
Davison J (Eds.), Models for Computer Aided Tolerancing in Design and Manufacturing, Springer, Netherlands, 2007, pp. 85-94.

[14]K. Jiang, J.K. Davidson, J. Liu, et al. Using tolerance maps to validate machining tolerances for transfer of cylindrical datum in manufacturing process, Int. J. Adv. Manuf. Technol. 73 (2014) 465-478.

[15]N. Anwer, B. Schleich, L. Mathieu, et al. From solid modeling to skin model shapes: shifting paradigms in computer-aided tolerancing, CIRP Ann. Manuf. Technol. 63 (2014) 137-140.

[16]M. Paulic, T. Irgolic, J. Balic, et al. Reverse engineering of parts with optical scanning and additive manufacturing, Procedia Eng. 69 (2014) 795-803.

[17]ISO 14405-1:2016, Geometrical product specifications (GPS)—Dimensional tolerancing— Part 1: linear sizes.

[18]ISO 17450-3:2016, Geometrical product specifications (GPS)—General concepts_ Part 3: Tolerance features.

Zhemin Tang is a Ph.D. candidate of Guilin University of Electronic Technology and has published three papers, all of which have already been indexed by EI. He is currently pursuing research in the field of computer-aided tolerance design and evaluation.

Meifa Huang is a professor and Ph.D. supervisor of Guilin University of Electronic Technology. He received his Ph.D. degree in 2004 from Huazhong University of Science and Technology, and he has published over 100 papers, including 53 papers indexed by SCI or EI. He is currently pursuing research in the field of smart measurement methods and precision design of electromechanical systems.

Yonghou Sun is a professor and Master supervisor of Guilin University of Electronic Technology, and he received his Master's degree in 1998 from China Agricultural University. He is currently pursuing research in the fields of modern design and manufacture, fluid transmission and control technology, fault diagnosis expert systems, and new generation GPS systems.

Yanru Zhong is a professor.of Guilin University of Electronic Technology. Her main research fields are software engineering and formalization methods, computer aided tolerance design, and a new generation of international precision theory systems, among others.

Yuchu Qin is currently a Ph.D. candidate at the EPSRC Future Advanced Metrology Hub, University of Huddersfield, UK. He received a D.Eng. degree in Measurement Technology and Instrument from the School of Mechanical Science and Engineering, Huazhong University of Science and Technology, China, in 2017. He has an M.Eng. degree in Computer Application Technology and a B.Eng. degree in Computer Science and Technology. His research interests lie in additive manufacturing informatics, computer-aided geometrical product specifications, CAD data interoperability, and knowledge representation and reasoning. He has published over ten papers about the representation of product and manufacturing information in several international journals, including Knowledge-Based Systems, Advanced Engineering Informatics, Computer-Aided Design, and Journal of Computing and Information Science in Engineering. He has also co-authored and published one academic monograph about knowledge representation of geometrical product specifications.

Jing Huang is a graduate student of Guilin University of Electronic Science and Technology. His main research fields are computer-aided tolerance design, and precision design and intelligent measurement, among others. 